\title{
Relación entre la tecno-procrastinación y el rendimiento académico: un estudio del caso
}

\section{Relationship between techno-procrastination and academic performance: a study of the case}

\author{
Cristina Ramón García \\ Universidad Miguel Hernández (España) \\ cristinarg223@gmail.com \\ Pedro Peinado Rocamora \\ Universidad Internacional de La Rioja (España) \\ pedro.peinado2@murciaeduca.es \\ María Elena Rodríguez Nieto \\ Consejería de Educación de la Región de Murcia (España) \\ mariaelena.rodriguez@murciaeduca.es
}

Recibido: 06/10/2020

Aceptado: $14 / 05 / 2021$

Publicado: 01/06/2021

\section{RESUMEN}

La gestión eficaz del tiempo es un aspecto esencial en los resultados académicos. El uso del teléfono móvil y su influencia en la adecuada administración del tiempo del estudiante configura el núcleo de este trabajo. La hipótesis de partida presupone una equivalencia negativa entre un excesivo uso del teléfono móvil y los resultados académicos. Para su verificación, elaboramos un índice de procrastinación (IP), normalizado posteriormente (IPN) para que tomara valores iguales o mayores que cero, calculado a partir del cuestionario utilizado como uno de los instrumentos de investigación. Partiendo de las 897 respuestas recabadas, enviadas desde 21 países, se ha procedido a su clasificación en seis bloques diferenciados, con el fin de facilitar su análisis. Por otra parte, para comprobar la correlación estadística existente entre las variables estudiadas, se ha diseñado otro cuestionario dirigido a alumnos del IES Los Albares, Cieza, de entre $3^{\circ}$ de la ESO y $2^{\circ}$ de Bachillerato que se ha cruzado con los datos de uso de sus teléfonos durante 352 semanas para 52 participantes. Tras el procesamiento y posterior análisis de la información obtenida, se ha comprobado que una puntuación elevada en cuanto a IPN complementada con un uso excesivo del teléfono móvil, ("tecno-procrastinación") no implica una disminución en el rendimiento académico. Por tanto, los resultados contradicen la hipótesis formulada.

\section{PALABRAS CLAVE}

Procrastinación; rendimiento académico; organización; TIC.

\section{ABSTRACT}

Effective time management is an essential aspect of academic results. The use of the mobile phone and its influence on the proper management of the student's time forms the core of this work. The starting hypothesis presupposes a negative equivalence between excessive use of the mobile phone and academic results. For its verification, we elaborated a procrastination index (PI), later normalized (NPI) to take values equal to or greater than zero, calculated from the questionnaire used as one of the research instruments. Based on the 897 responses collected, sent from 21 countries, they have been classified into six distinct blocks in order to facilitate their analysis. On the other hand, to verify the statistical correlation between the variables studied, another questionnaire has been designed for students of the IES Los Albares, Cieza, between the 3rd year of secondary school and the 2 nd year of high school, which has been crossed with the usage data of their phones during 352 weeks for 52 participants. After processing and subsequent analysis 
of the information obtained, it has been found that a high NPI score complemented by excessive use of the mobile phone ("techno-procrastination") does not imply a decrease in academic performance. Therefore, the results contradict the hypothesis formulated.

\section{KEYWORDS}

Procrastination, academic performance, organization, ICT.

\section{CITA RECOMENDADA}

Ramón, C., Peinado, P. \& Rodríguez, M.E. (2021). Relación entre la tecno-procrastinación y el rendimiento académico: un estudio de caso. Revista Interuniversitaria de Investigación en Tecnología Educativa, 10, 23-42. https://doi.org/10.6018/riite.449161

\section{Principales aportaciones del artículo y futuras líneas de investigación:}

- La descripción del uso de los dispositivos móviles, medido en tiempos y en aplicaciones predominantes.

- El diseño y cálculo de un Índice de tecno-procrastinación académica

- El cálculo de la correlación entre el uso del teléfono móvil (tecno-procrastinación) y las calificaciones académicas

\section{INTRODUCCIÓN}

En el presente trabajo se analiza la importancia que supone la gestión del tiempo en la organización de tareas y los resultados académicos de los estudiantes preuniversitarios dentro de la sociedad tecnológica en la que vivimos, así como la relevancia del fenómeno de la procrastinación en el ámbito académico.

Demorar la ejecución de una tarea no tiene por qué deberse simplemente a una actitud de holgazanería, también intervienen aspectos como una mala gestión del tiempo, o incluso factores psicológicos de mayor calado, entre los que se incluyen los trastornos de atención. Existe procrastinación en cualquier contexto: profesional, académico o de la vida cotidiana. Sin embargo, debido al alcance del fenómeno en el sector académico, este trabajo se centrará en valorar y analizar la procrastinación en dicho ámbito.

El uso de los teléfonos móviles por los estudiantes se ha ido convirtiendo en un tema controvertido socialmente, hasta plantear desde las administraciones educativas su prohibición en las aulas. Resulta de interés averiguar si el uso de estos dispositivos repercute en el desempeño de las tareas, siendo el problema de investigación planteado: ¿existe correlación entre el tiempo de uso de los teléfonos móviles, la procrastinación académica y el rendimiento académico?

Para delimitar el tema, nos hemos permitido una licencia lingüística proponiendo un término de libre composición, "tecno-procrastinación", entendido como la relación de causa-efecto entre el uso del teléfono móvil, y, por extensión, de las nuevas tecnologías, internet y las redes sociales, y la procrastinación.

\section{ESTADO DEL ARTE}

\subsection{Procrastinación: Concepto y uso del término}

Según Klassen et al. (2008), las primeras referencias sobre "procrastinación" remontan al siglo XIX. Sin embargo, el término ya aparece registrado documentalmente en Francia en el siglo XVI (Robert, 1972), convirtiéndose en objeto de estudio en el siglo XX. 
En la siguiente tabla (Tabla 1) se recogen las principales aportaciones para definir el término:

Tabla 1. Aspectos centrales recurrentes en la definición de procrastinación

\begin{tabular}{|c|c|}
\hline Autor & Aspectos centrales \\
\hline Solomon \& Rothblum (1994) & $\begin{array}{c}\text { Predisposición para completar la tarea atrasada que se da en } \\
\text { personas emocionalmente tristes. }\end{array}$ \\
\hline Senecal et al. (1995) & Problema de organización individual. \\
\hline Ferrari (2000) & Incapacidad de mantener la atención en una sola tarea. \\
\hline Steel (2007) & Fracaso autorregulatorio que no se comprende por completo. \\
\hline Angarita (2012) & $\begin{array}{l}\text { Aplazamiento voluntario de tareas académicas por otras que } \\
\text { pueden mantener relación o no con el ámbito académico, } \\
\text { conociendo las consecuencias. }\end{array}$ \\
\hline Cano et al. (2016) & Acción de retrasar tareas que deben ser atendidas. \\
\hline
\end{tabular}

Teniendo en cuenta todas las referencias citadas, la procrastinación puede definirse como una acción o comportamiento puntual o habitual, que implica el aplazamiento o atraso de la realización de una tarea, debido a diferentes factores externos.

\subsection{Procrastinación académica}

La procrastinación puede extenderse a cualquier ámbito, sin embargo, en algunos de ellos es susceptible de ser más perjudicial que en otros. Como caso paradigmático de ambiente proclive a la procrastinación, aparece el contexto académico, donde es un comportamiento muy frecuente según Rosario et al., (2009). Además, para Uzun et al. (2014), la procrastinación es una de las barreras comunes que aumenta en el sector académico y Álvarez (2010) asegura que está vinculada con notas bajas y el fracaso escolar.

Asimismo, Dewitte \& Schouwenburg (2002) presentaban la incapacidad de los más jóvenes para rechazar tentaciones y distracciones como una eventual explicación del incremento de la procrastinación académica en la adolescencia, e investigaciones como la de Rodríguez \& Clariana (2017) confirman que el fenómeno se intensifica progresivamente hasta llegar a Bachillerato.

\subsection{Adicción a internet: Tecno-procrastinación}

Actualmente el uso de las tecnologías de información y de Internet se extiende progresivamente en la población adolescente $y$, pese a constituir una fuente eficaz de información y de interacción social, su uso excesivo puede conllevar riesgos de dependencia (Toro, 2010).

Quan-Haase \& Wellman (2005) definen la hiperconectividad como la disposición para comunicarse en todo momento, a través del empleo del móvil o el computador. Sin embargo, refirieron sus efectos negativos en la concentración, imposibilitando la realización de tareas. Precisamente, los estudiantes universitarios manifestaron que cuando están frente al ordenador para trabajar, permanecen en alerta para recibir y enviar mensajes.

Reig \& Vílchez (2013) apuntan que la hiperconectividad constituye una nueva era, caracterizada por la permanente conexión de las personas en las pantallas táctiles que visualizan imágenes en constante movimiento o conversan continuamente, y Pinto \& Díaz (2015) alertan sobre los usos excesivos de tecnología digital y sus posibles consecuencias, considerando la necesidad de acompañar al adolescente en el uso del mundo digital. Aunque no es una línea abundantemente explotada por la literatura científica, investigaciones como la de Ugaz \& Lizana (2019) relacionan directamente la procrastinación académica y la dependencia al móvil, lo que se ha denominado en este trabajo "tecno-procrastinación". 


\section{MÉTODO}

La metodología de investigación de este estudio emplea un enfoque cuantitativo con alcance correlacional de proceso polietápico y método mixto de recogida de información, dado que, para llevar a cabo la toma de datos han sido utilizados tanto instrumentos cualitativos como cuantitativos.

Además, es un estudio con doble naturaleza: transversal por la ejecución del cuestionario, y longitudinal por el seguimiento durante 153 días (5 meses) del tiempo de uso de los dispositivos móviles y de las aplicaciones consultadas.

\subsection{Hipótesis y objetivos}

La hipótesis de partida de esta investigación es que el uso excesivo del teléfono móvil es un factor relevante en la procrastinación (tecno-procrastinación) y afecta negativamente en el rendimiento académico.

A partir de la hipótesis propuesta, se elaboraron los siguientes objetivos de investigación: Como objetivo general:

Comprobar la relación entre la tecno-procrastinación en estudiantes de enseñanzas secundarias y universitarias, y su rendimiento académico.

Siendo los objetivos específicos:

1. Analizar el grado de procrastinación existente en el alumnado de enseñanzas medias y superiores.

2. Establecer una correlación entre el grado de procrastinación del alumnado, el tiempo de uso de dispositivos móviles y sus calificaciones.

\subsection{Contexto y participantes}

La intervención se desarrolló en un doble contexto, virtual y presencial. En una primera fase, se recolectaron datos a través de Google Forms (exclusivamente online) con la Muestra I, puesto que la pretensión era tener representatividad tanto nacional como internacional. La segunda fase se realizó únicamente con alumnado del IES Los Albares (Cieza, Murcia), Muestra II, por la necesidad de efectuar el seguimiento del uso de dispositivos y calificaciones de los sujetos seleccionados.

El total de participantes en el estudio fue de 949 estudiantes dividido en dos muestras:

La muestra I está formada por 897 estudiantes provenientes de 21 países con edades comprendidas entre los 13 y 27 años, distintos niveles de estudios (ESO, bachillerato y estudios universitarios) y con una distribución de sexos de un $66 \%$ de mujeres y $34 \%$ de hombres. Dicha muestra se obtuvo a partir de un muestreo no probabilístico de bola de nieve, en el que cada sujeto propone a otros provocando así un efecto acumulativo que simula una bola de nieve.

La muestra II se compone de 52 alumnos de entre $3^{\circ}$ ESO y $2^{\circ}$ Bachillerato del IES Los Albares. Su elección se llevó a cabo a partir de un muestreo por conveniencia de alumnado del IES Los Albares que poseyeran un teléfono móvil de la marca Apple y con los que pudiera mantenerse contacto diario.

Inicialmente, la muestra II comenzó con 18 alumnos de Bachillerato de Investigación, que componen la submuestra IIA. Posteriormente, en una segunda etapa de recogida de datos se aumentó dicha muestra, sumando 34 alumnos más que conforman la submuestra IIB, con alumnos que no pertenecían a este programa de especificación curricular, teniendo 30 de ellos al menos una asignatura suspensa. El objetivo de esta ampliación fue hacer la muestra más real y representativa, ya que la muestra II original (submuestra IIA) estaba compuesta exclusivamente por alumnado sin asignaturas suspensas, común en el programa de Investigación. 


\subsection{Instrumentos}

El primer instrumento se completó en dos etapas, aplicando un cuestionario con dos versiones. Ambas contaban con un bloque común, ya que comparten el mismo objeto de estudio; sin embargo, en la segunda versión se añadió una parte adicional de datos personales con la intención de obtener correlaciones con el resto de parámetros recopilados en este trabajo.

En primer lugar, se realizó una primera versión del cuestionario dirigido a la Muestra I $(n=897)$, redactado en tres versiones: español, inglés y francés. Se llevó a cabo de manera anónima y confidencial, recogiendo datos cualitativos a través de una escala numérica con el objeto de establecer comparaciones y relaciones.

Por otra parte, la segunda versión se destinó a la Muestra II $(n=52)$. Sus participantes aportaron datos personales como su nombre y apellidos, número regional de estudiante o DNI, para poder acceder a sus calificaciones y así establecer una correlación entre el eventual grado de procrastinación que revelasen las respuestas de cada encuestado y sus resultados académicos.

Tabla 2. Datos generales del cuestionario

\begin{tabular}{ccc}
\cline { 2 - 3 } & $\begin{array}{c}\text { Cuestionario } \\
\text { Versión 1 }\end{array}$ & $\begin{array}{c}\text { Cuestionario } \\
\text { Versión 2 }\end{array}$ \\
\hline $\begin{array}{c}\text { № de participantes } \\
\text { № de bloques }\end{array}$ & 897 & 52 \\
№ de ítems & 6 & 7 \\
$\begin{array}{c}\text { Bloques de estudio } \\
\text { comunes }\end{array}$ & 22 & 23 \\
& Conocimiento término procrastinación \\
Planificación del tiempo \\
Gestión del tiempo
\end{tabular}

Con las dos versiones se realizó un pretest para conocer su adecuación y validez. Los datos recogidos fueron cualitativos, obtenidos a partir de la valoración de cada ítem en una escala de 0 a 10 (siendo 0 nada de acuerdo y 10 muy de acuerdo), excepto 4 de las cuestiones, que eran de opción múltiple. Además, para conseguir correlacionar los resultados y dar respuesta a los objetivos planteados, se elaboró un Índice de Procrastinación (IP), que será desarrollado en el apartado 4.

El segundo instrumento consistió en la recogida y análisis de datos de los registros de uso del teléfono móvil (marca Apple) de cada participante procedente de la Muestra II. Se realizó a partir de la información semanal de la aplicación "Tiempo de uso" (véanse en el apartado 4) entre septiembre 2019 y febrero 2020.

Por último, el tercer instrumento utilizado fue el registro de las calificaciones académicas del primer trimestre del curso 2019/2020 de los alumnos de la Muestra II, cuya utilidad consiste en comprobar si procrastinar puede tener repercusión en las calificaciones del alumnado de la muestra y, por tanto, ser relevante o no en su rendimiento académico. 


\subsection{Fases de la investigación}

El trabajo se ha dividido en las diferentes fases (Figura 1):

\section{Fase preparatoria}

Diseño de la investigación y revisión bibliográfica.

Definición del diseño de la metodología y los instrumentos.

\section{Trabajo de campo}

Aplicación de los instrumentos de investigación en 3 etapas

Etapa 1: Cuestionario nacional e internacional (Instrumento 1)

Etapa 2: Recopilación datos usos teléfonos (Instrumento 2)

Etapa 3: Recopilación de calificaciones (Instrumento 3)

Organización de datos.

\section{Fase analítica}

-Análisis de datos.

- Obtención de conclusiones.

- Elaboración del documento final.

Figura 1. Fases de la investigación

Como la aplicación de los instrumentos es la parte más extensa y significativa ahondaremos en ella. Consiste en un periodo polietápico con la siguiente división:

- Durante la Etapa 1 (junio-noviembre 2019) se elaboró el cuestionario. En primer lugar, se confeccionó el modelo básico que se adaptó a los idiomas de inglés y francés para conseguir un alcance más amplio, obteniendo finalmente 897 respuestas procedentes de 21 países de todo el mundo (Alemania (5), Argelia (1), Argentina (1), Bulgaria (5), Colombia (28), Dinamarca (2), España (761), EEUU (6), Finlandia (9), Francia (57), Guinea (1), Hungría (1), Irlanda (8), Japón (4), Marruecos (1), Noruega (1), Perú (1), Polonia (1), Reino Unido (1), Rusia (1), Suiza (3)).

Más tarde, se adaptó la segunda versión del cuestionario con el fin de recabar los datos necesarios para realizar la correlación entre el IPN de la Muestra II y sus calificaciones académicas.

- En la Etapa 2 (septiembre 2019-febrero 2020), desde el 9/9/19 hasta el 9/2/20 (153 días), se recopilaron datos del uso del teléfono móvil procedentes de 52 alumnos de diferentes cursos académicos. Se analizaron un total de 352 semanas, que suponen 2464 días o 59136 horas de uso de los dispositivos móviles.

- En la Etapa 3 (enero-febrero 2020), se recopilaron las calificaciones académicas de la Muestra II y se calcularon las correlaciones entre el IPN y el tiempo de uso del teléfono móvil.

\section{RESULTADOS}

\subsection{Cálculo de IPN}

Como eje central de la investigación, se confeccionó el índice a partir del cuestionario completado por las dos muestras fijadas. Su definición matemática quedó vertebrada en tres sumandos:

$$
\text { IPN }=\sum \text { preguntas positivas }+\sum \text { preguntas negativas }+\mathbf{m}
$$

En un principio, el Índice de Procrastinación (IP) quedó definido por los dos primeros sumandos. El primero, compuesto por las puntuaciones agregadas de las 13 preguntas que 
aportan valores positivos (no constituyen acciones procrastinadoras), y el segundo término, compuesto por las puntuaciones agregadas de las 8 preguntas que aportan valores negativos (constituyen acciones procrastinadoras), pero, para facilitar su entendimiento, se normalizó matemáticamente situando el mínimo valor del índice en 0 a través de un tercer término en la fórmula, la suma del valor mínimo del IP inicial $(m=-54,5)$ obtenido en la muestra.

El resultado debe interpretarse de tal forma que, cuanto mayor puntuación arroje el Índice de Procrastinación Normalizado (IPN), menor será el grado de procrastinación del encuestado, obteniéndose el mínimo muestral en 0 y el máximo en 176,5.

\subsection{Instrumento 1: Cuestionario}

Las respuestas de este cuestionario se dividieron en 6 bloques (Figura 2):

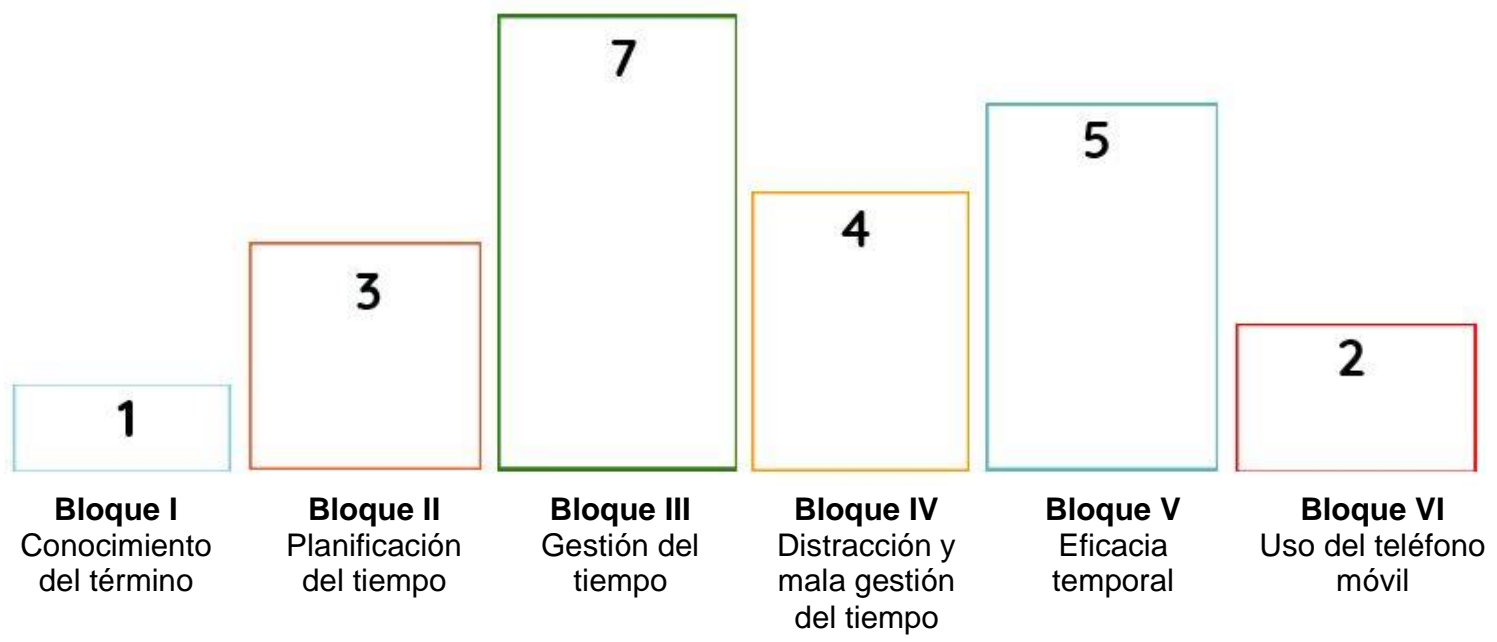

Figura 2. División en bloques del cuestionario

El Bloque I consta de una única cuestión y muestra el conocimiento del término "procrastinación" a partir de la elección entre tres afirmaciones. Como se comprueba en la Figura 3 , aproximadamente 3 de cada 4 encuestados contestaron de manera correcta a esta pregunta.

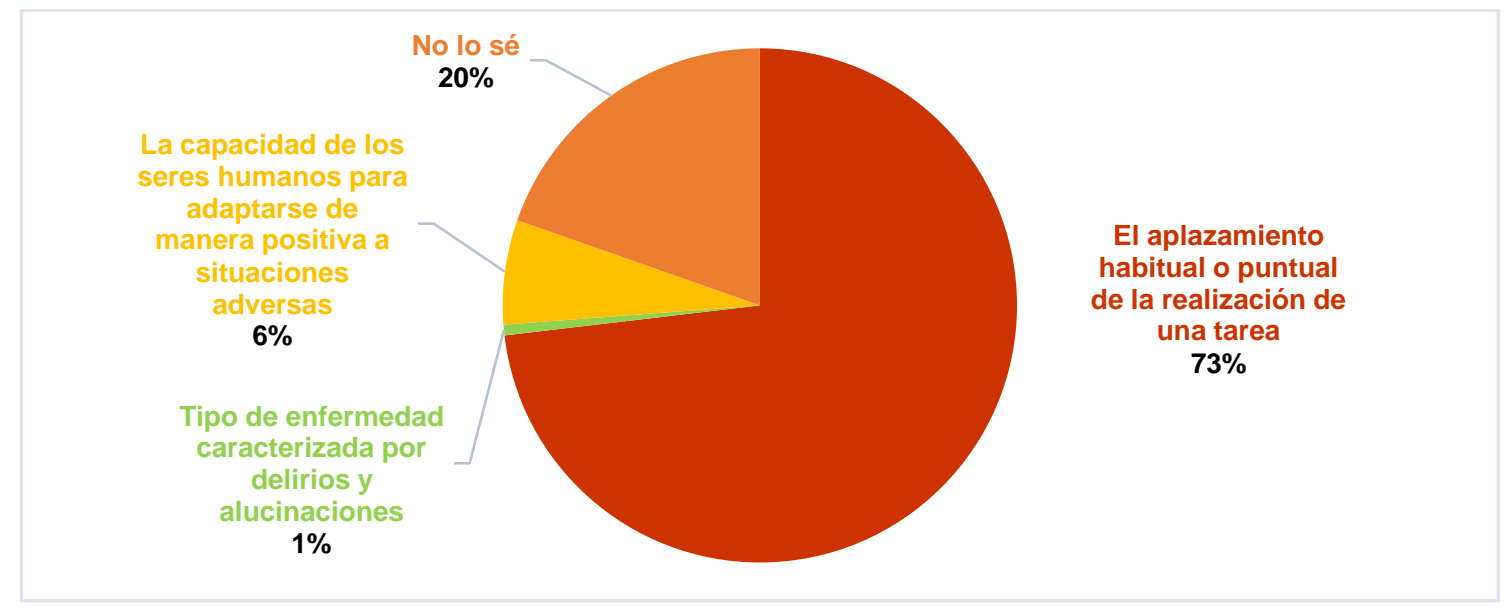

Figura 3. Respuestas a la pregunta 1 sobre la definición del término procrastinación

Dentro del segundo bloque, se propusieron 3 afirmaciones que presentan evidentes diferencias en sus promedios de puntuación. Así, los datos de la Figura 4 nos muestran que es habitual tener una agenda o un calendario, pero es menos frecuente contar con planificadores de estudio. 


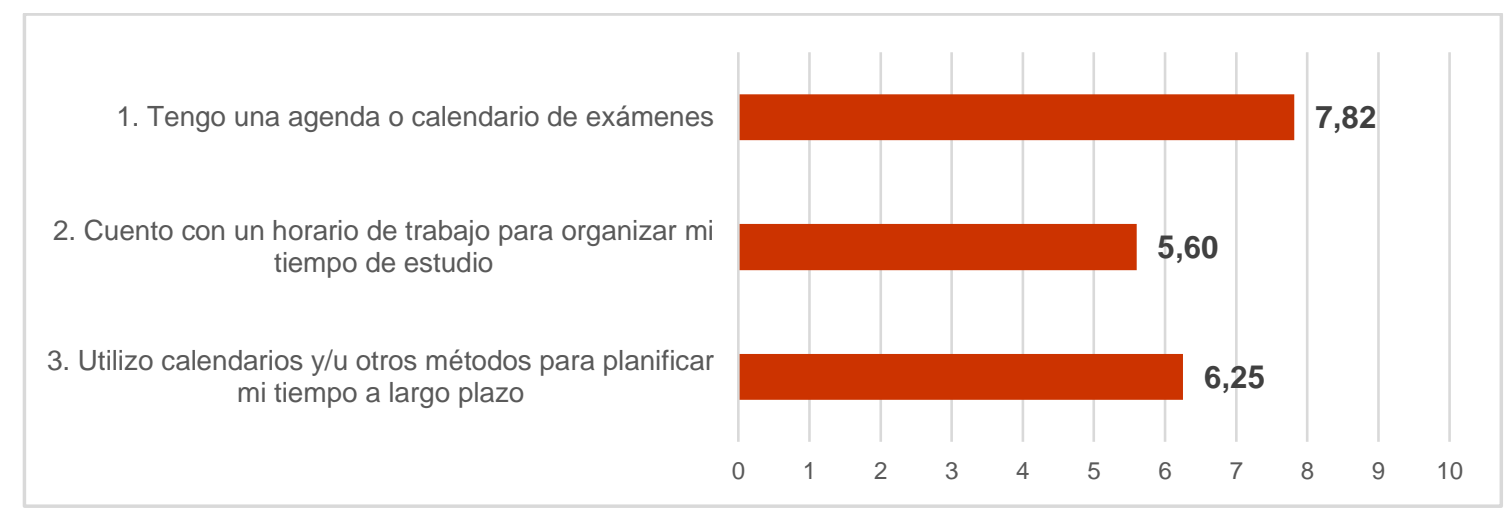

Figura 4. Resultados de las cuestiones del Bloque II

Por otra parte, dentro del Bloque III podemos encontrar cuestiones de carácter cualitativo y cuantitativo. Con respecto a la Figura 5, se observa una clara disparidad entre las diferentes opciones, obteniendo un mayor número de elecciones (casi un $35 \%$ ) la opción que no implica procrastinar.

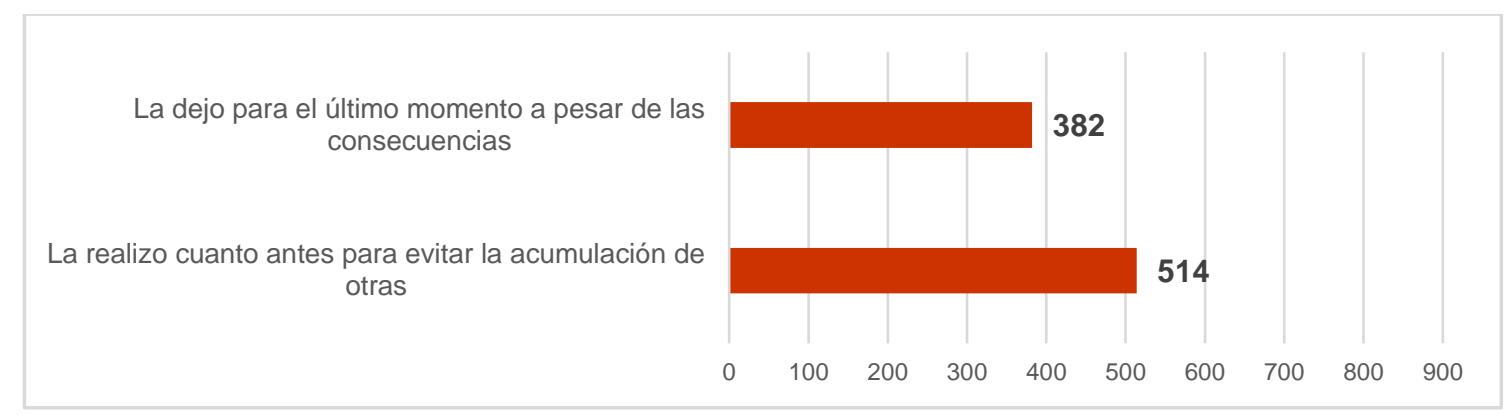

Figura 5. Resultados de la pregunta: "Cuando me mandan una tarea a largo plazo"

Asimismo, en la Figura 6 podemos comprobar que la media de respuestas entre la primera y la segunda afirmación son prácticamente similares, mientras que la tercera afirmación tiene una media de respuestas levemente menor que las dos anteriores.

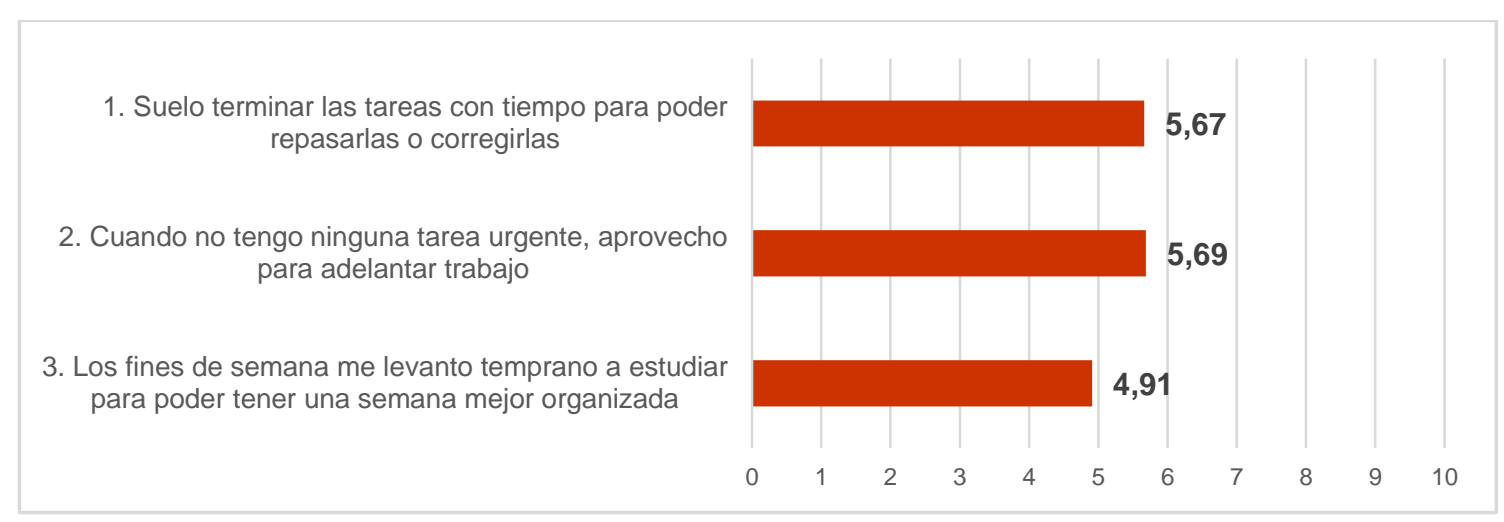

Figura 6. Resultados de las cuestiones del Bloque III

Seis de las siete afirmaciones del Bloque IV (a excepción de la 7) incluidas en la Figura 7 puntúan negativamente en el IPN, lo que supone un incremento del nivel de procrastinación, ya que, a menos puntuación del IPN, más procrastina el encuestado. Prácticamente todos los valores se mueven en valores centrales del rango (aproximadamente entre 5 y 7 ) lo que muestra moderación en todos los indicadores del bloque. 
1. Mis padres o personas cercanas de mi entorno me dicen que pierdo mucho el tiempo

2. Suelo acostarme tarde y, en consecuencia, me levanto tarde y voy con prisas para ir a clase

3. Muchas veces pierdo el tiempo hablando por el móvil con mis amigos de temas sin importancia

4. Reviso aplicaciones en el móvil reiteradamente tras haberlas consultado hace poco tiempo

5. Suelo ocupar parte del tiempo destinado a mis obligaciones con otras actividades de ocio (por ejemplo jugar, entrar en RRSS...)

6. Cuando estoy estudiando, tengo el móvil en la misma habitación y suelo distraerme

7. Cuando estoy estudiando, me centro solo en eso y evito distraerme con otros quehaceres

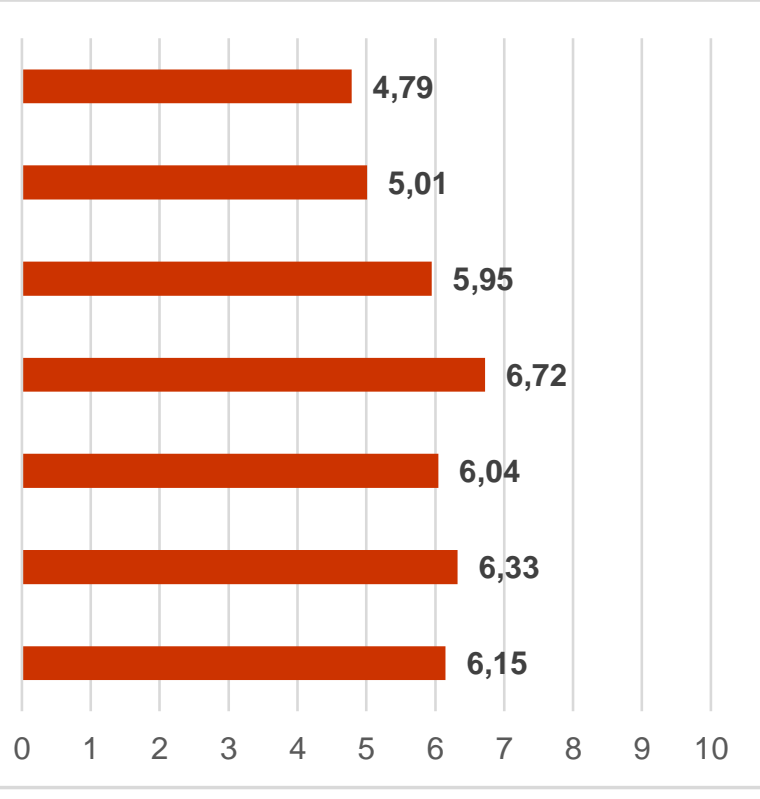

Figura 7. Resultados de las cuestiones del Bloque IV

Dentro del bloque V, Figura 8, contamos con cinco afirmaciones agrupadas en dos apartados. En el primero, se observa la eficacia que tiene el alumnado con respecto a su gestión del tiempo. Tres de ellas (1, 2 y 4 ) puntúan de manera positiva en el IPN. Las afirmaciones 2 y 4 han obtenido un promedio de puntuación mayor que 7 , mientras que la 1 no llega a 6 puntos de promedio, siendo la puntuación más baja del bloque. Por otra parte, la otra afirmación (3) supone una puntuación negativa y su promedio de puntuación supera los 6 puntos.

1. Cuando organizo un horario de estudio lo cumplo sin modificaciones ni excepciones

2. Cuando planifico mi trabajo, obtengo mejores resultados

3. Algunas veces me centro tanto en ciertas asignaturas que acabo dejando otras de lado

4. Cuando una asignatura me resulta más difícil que otras, procuro dedicarle más tiempo

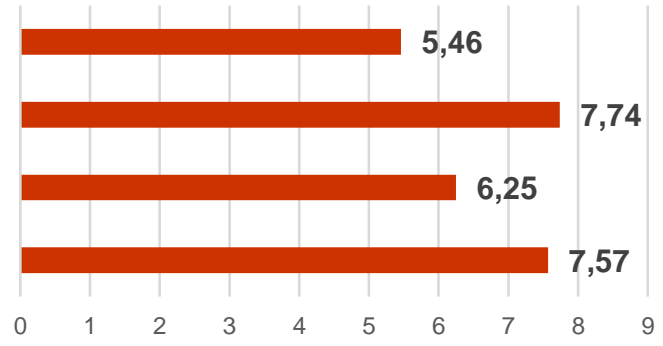

Figura 8. Resultados de las cuestiones del Bloque V

Se destinó una afirmación, que se muestra en la Figura 9, a comprobar si la procrastinación activa se encontraba presente en los estudiantes, entendiéndose como procrastinación activa aquella que es realizada por los procrastinadores "positivos", es decir, los que posponen la ejecución de sus tareas porque prefieren trabajar bajo presión o toman decisiones deliberadas para poder posponerlas (Chu \& Choi, 2005).

Se observa una clara diferencia con respecto a la mínima puntuación frente a las demás. Se concentra un 30,3\% de encuestados en 1 (totalmente de acuerdo), mostrando que buena parte de ellos no estudia normalmente solo el día antes del examen, además, un $65 \%$ seleccionó una puntuación de 50 inferior. Por otra parte, un 7\% del total eligió la máxima puntuación 10, totalmente en desacuerdo). 


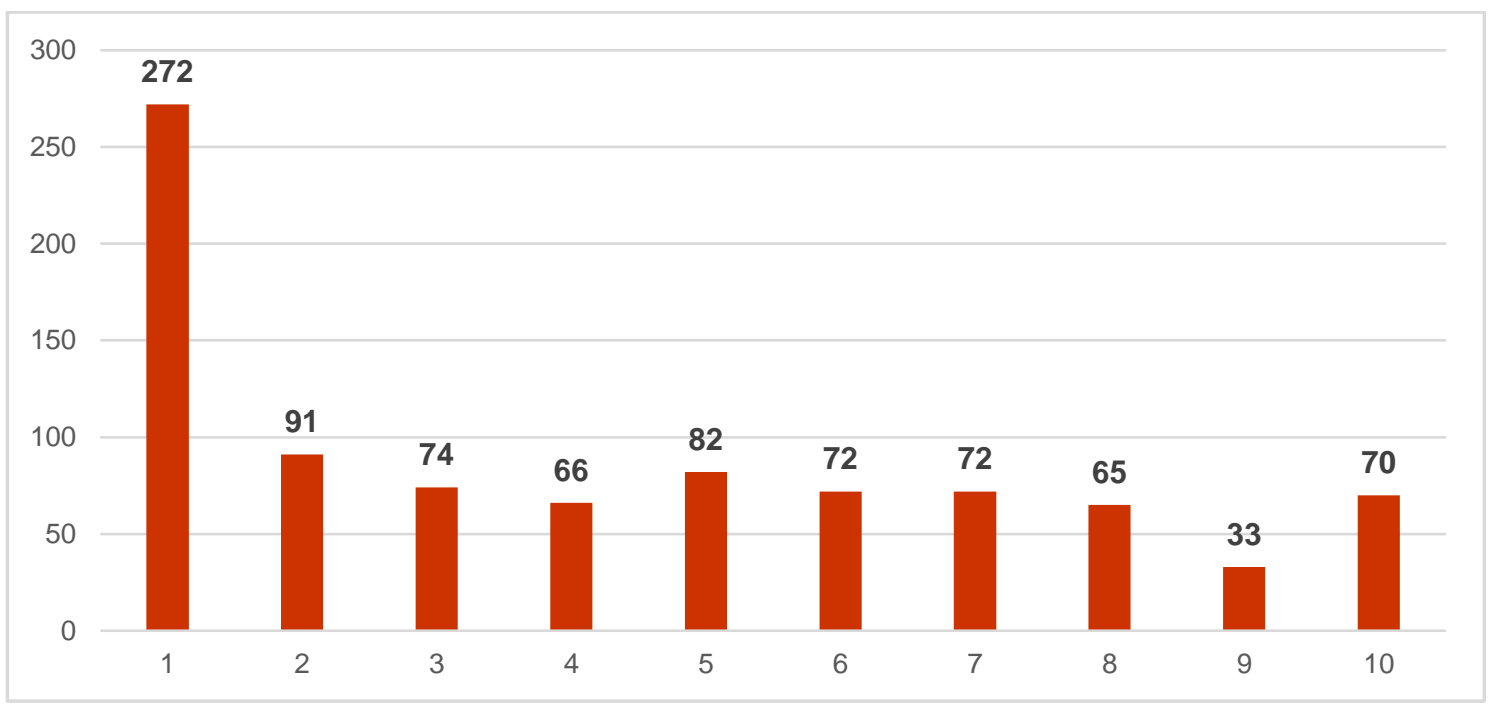

Figura 9. Resultados de la pregunta: "Normalmente estudio solo el día antes del examen porque suelo trabajar mejor bajo presión"

En el Bloque VI, Figura 10a, se muestra cómo 544 encuestados afirman conectarse a las redes sociales entre 20 y 100 veces al día, siendo la opción claramente destacada en esta pregunta. Y, en la Figura 10b, la única opción desigual es la de menos de 1 hora de uso de móvil diario (26 encuestados), quedando las otras tres opciones con porcentajes más homogéneos, siendo la opción de uso de móvil más de 3 horas diarias la de mayor frecuencia, con 340 respuestas.

Conjugando ambos datos puede extraerse que, en el contexto de este trabajo, un estudiante tipo se conecta como mínimo entre 1 y 2 horas diarias y entre 20 y 100 veces a WhatsApp.

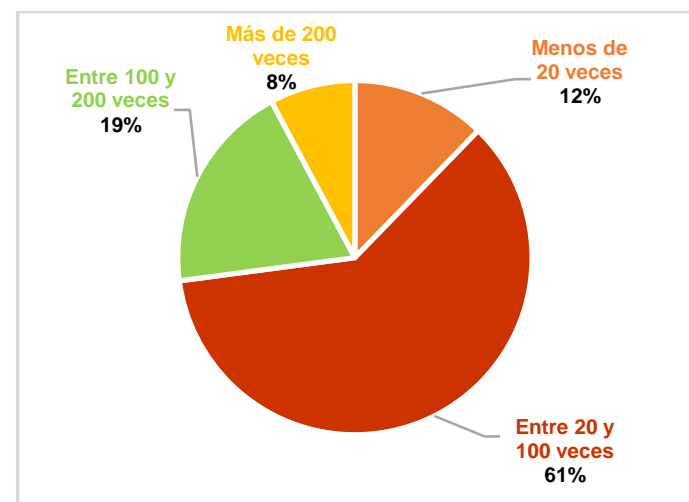

Figura 10a. Resultados para la pregunta "Al día me conecto a las RRSS y aplicaciones de mensajería (WhatsApp)"

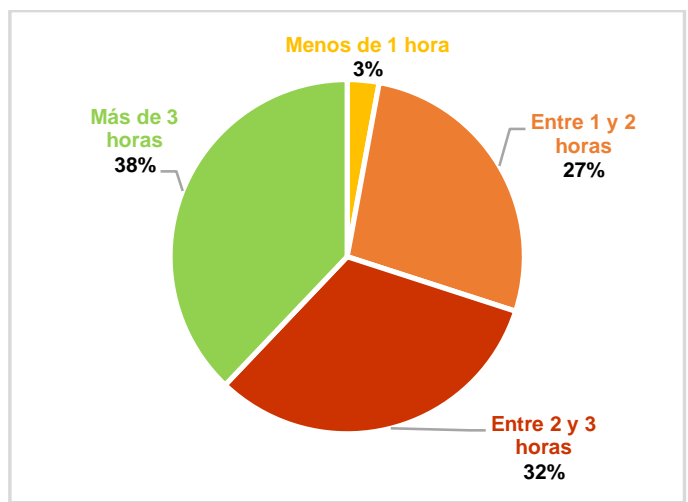

Figura 10b. Resultados para la pregunta "La media de horas diarias que paso utilizando el teléfono móvil se sitúa en:"

En la Tabla 3 se muestran las medias de todos los bloques y el valor mínimo muestral. Excepto el Bloque IV, todos tienen contribución positiva al IPN. Se ha incluido en la tabla el mínimo con signo positivo ya que es el factor que normaliza el índice de procrastinación. 
Tabla 3. Media parcial IPN por bloques

\begin{tabular}{cc}
\cline { 2 - 2 } & Media parcial IPN \\
\hline Bloque I & 7,57 \\
Bloque II & 6,56 \\
Bloque III & 4,99 \\
Bloque IV & $-4,1$ \\
Bloque V & 3,63 \\
Bloque VI & 4,95 \\
Mínimo & 54,5 \\
Sumatorio de las medias parciales & 78,1 \\
\hline
\end{tabular}

Una vez calculados los parámetros descriptivos más relevantes de la muestra, es importante destacar varias características que refuerzan la validez y la confiabilidad del diseño y resultados del IPN. Por un lado, la igualdad de facto de la media $(88,07)$ y la mediana $(89)$, y por otro, el coeficiente de asimetría $(-0,12)$ y el coeficiente de curtosis $(-0,48)$, ambos prácticamente cero, revelan la existencia de una distribución normal casi ejemplar, además, muestran una función mesocúrtica propia de estas distribuciones (Tabla 4).

Tabla 4. Resumen de los parámetros estadísticos

\begin{tabular}{cc}
\hline Media & 88,0774805 \\
Error típico & 1,06279937 \\
Mediana & 89 \\
Moda & 109,5 \\
Desviación estándar & 31,8307968 \\
Varianza de la muestra & 1013,19963 \\
Curtosis & $-0,48025364$ \\
Coeficiente de asimetría & $-0,12373099$ \\
Rango & 176,5 \\
Mínimo & 0 \\
Máximo & 176,5 \\
Suma & 79005,5 \\
Cuenta & 897 \\
\hline
\end{tabular}

Se puede observar también la distribución de percentiles acumulada (Figura 11), en la que ambas colas crecen de forma paralela y el $80 \%$ central lo hace con el crecimiento sostenido típico de esta función Normal.

Observando el IPN obtenido de la muestra global (Figura 12), podemos comprobar que sigue fielmente la distribución normal descrita (campana de Gauss) en la que la mayoría de los encuestados se encuentran en el intervalo central de entre 80 y 100 puntos, siendo el promedio de IPN de la muestra 88,07. 


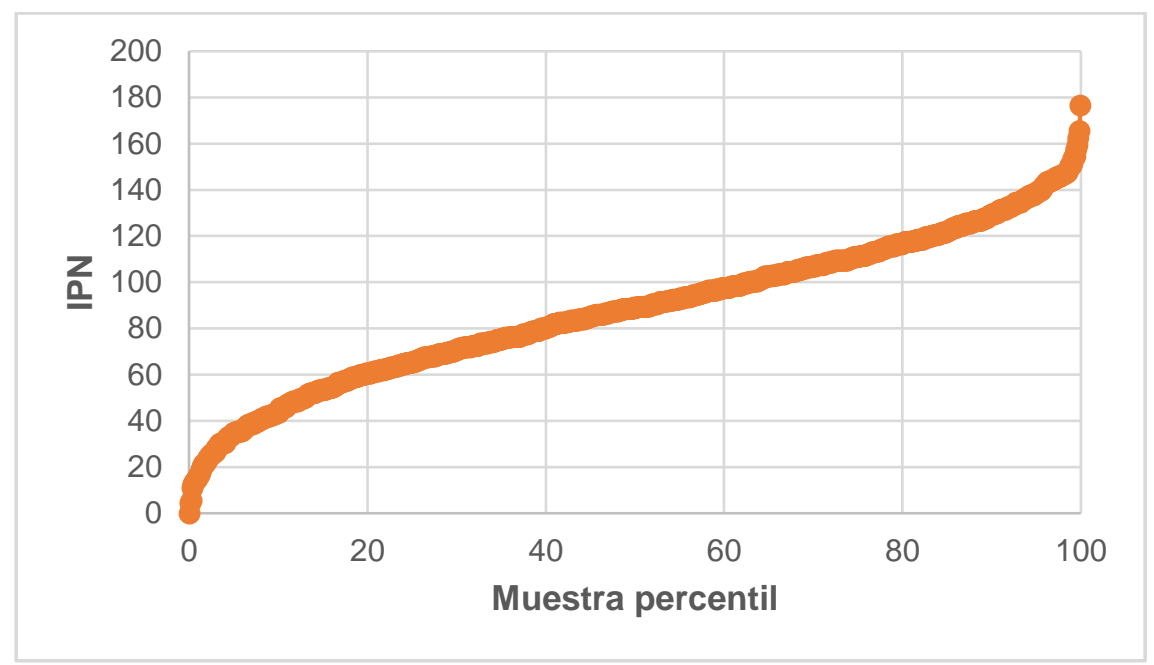

Figura 11. Distribución acumulada de percentiles del IPN

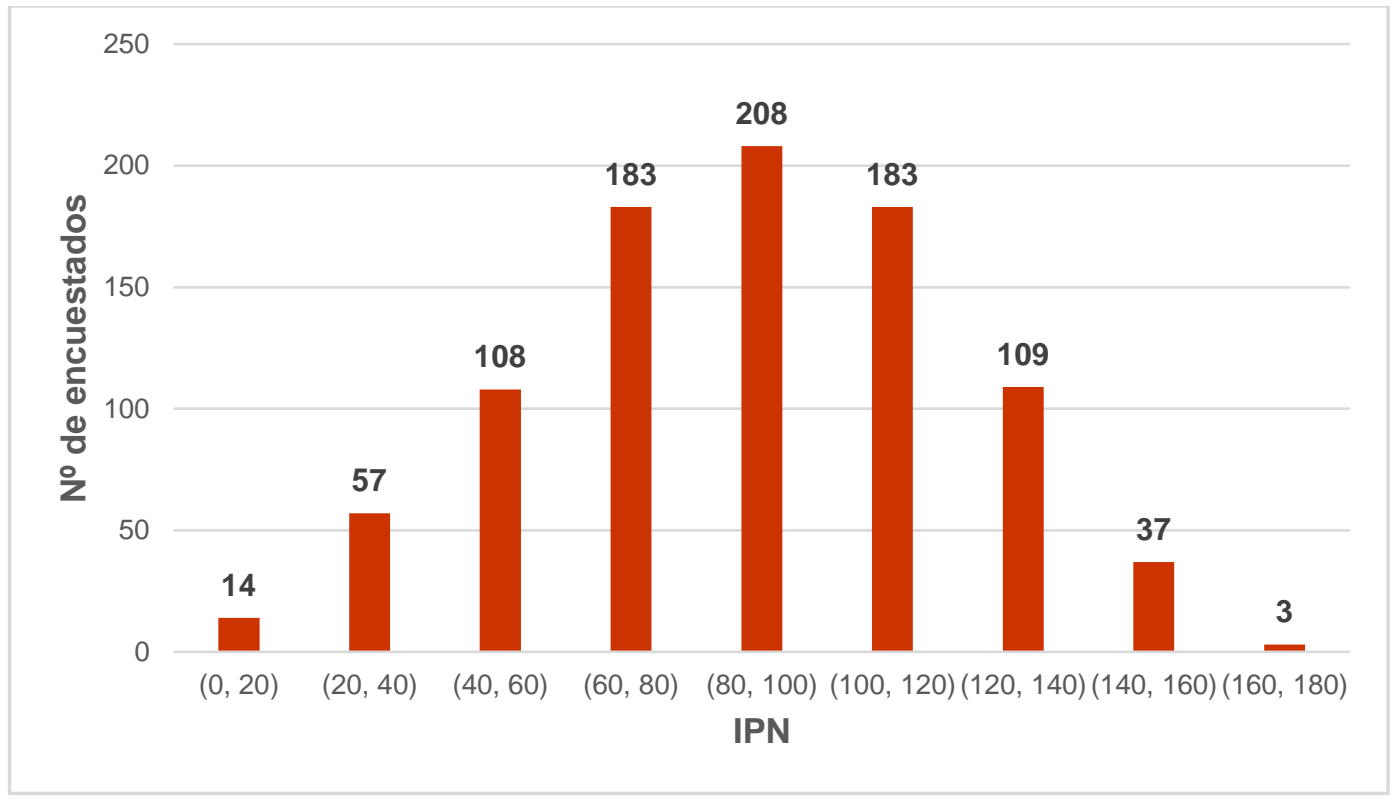

Figura 12. Encuestados - IPN global

Dividiendo la muestra por sexos (Figura 13), podemos observar que las mujeres ( $n=593$ ) presentan un IPN de media 91,43 puntos, casi 10 puntos por encima de la media de los hombres $(n=304)$, que se sitúa en 81,53 , y 3 puntos superior a la media de IPN total $(88,07)$, siendo el máximo muestral 176,5 y el mínimo muestral 0 , como ya se ha apuntado en apartados anteriores. 


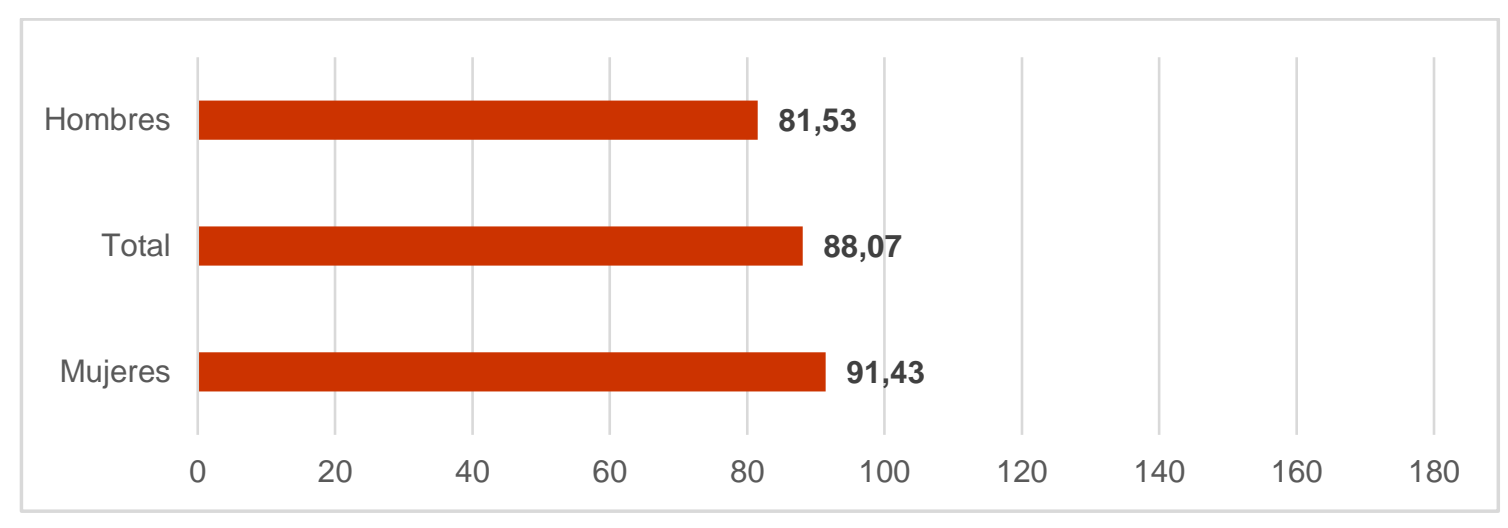

Figura 13. IPN por sexo

\subsection{Instrumento 2: Registros de uso del teléfono móvil}

Los registros de uso se recogieron a partir de capturas de pantalla como las que se exponen en las siguientes figuras (Figura 14a y Figura 14b) en las que aparecen datos de la media del tiempo de uso del teléfono móvil de uno de los alumnos seleccionados en la submuestra.

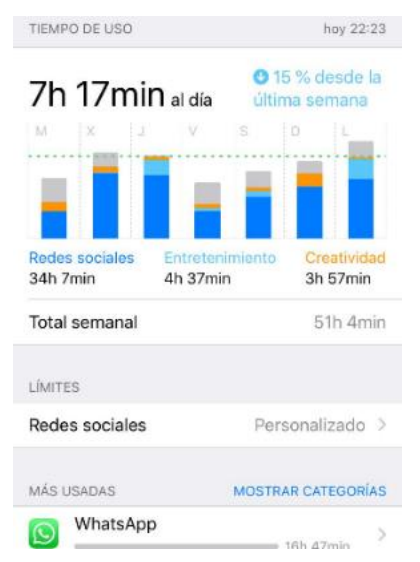

Figura 14a. Captura tiempo de uso

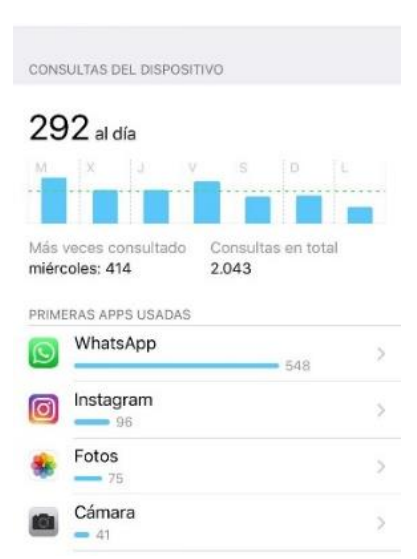

Figura 14b. Captura consultas app

La Figura 14a, en la que se sitúa la primera captura, nos permite obtener datos acerca de la media de uso al día durante una semana, el tiempo empleado en redes sociales, en las aplicaciones destinadas al entretenimiento y en las destinadas a la creatividad, aunque esto varía en función de cada sujeto.

La Figura 14b ofrece datos acerca de la media de las consultas diarias durante una semana, así como las consultas diarias de cada una de las aplicaciones más usadas; de las cuales solo se extrajeron aquellos datos pertenecientes a las tres primeras.

De estas dos fuentes de datos semanales de cada participante se recopilaron 352 semanas de 52 alumnos (generando así un total de 2464 días, 59136 horas evaluadas). Debido a la ingente cantidad de datos, se presenta en la Tabla 5 un resumen de los parámetros estadísticos pertenecientes a este instrumento. 
Tabla 5. Resumen registro de datos

\begin{tabular}{|c|c|c|c|c|c|}
\hline Parámetro & Mínimo & Máximo & Media $(\mu)$ & $\begin{array}{c}\text { Desviación } \\
\text { típica }\left(\mathrm{S}_{\mathrm{x}}\right)\end{array}$ & $\begin{array}{c}\text { Intervalo } \\
\text { característico } \\
\left(\mu-S_{x}, \mu+S_{x}\right)\end{array}$ \\
\hline $\begin{array}{c}\text { Minutos } \\
\text { semanales en } \\
\text { RRSS }\end{array}$ & 382,67 & 2295,75 & 1195,79 & 400,968 & $(794,82-1596,75)$ \\
\hline $\begin{array}{l}\text { Minutos } \\
\text { totales de } \\
\text { uso del móvil }\end{array}$ & 942,50 & 3300,50 & 2121,5 & 1667,36 & $(454,14-3788,86)$ \\
\hline $\begin{array}{c}\text { Promedio } \\
\text { semanal de } \\
\text { entradas App } \\
1\end{array}$ & 108,86 & 559,75 & 277,60 & 98,063 & $(179,54-375,66)$ \\
\hline $\begin{array}{c}\text { Promedio } \\
\text { semanal de } \\
\text { entradas App } \\
2\end{array}$ & 50 & 330,8 & 141,10 & 62,127 & $(78,97-203,23)$ \\
\hline $\begin{array}{c}\text { Promedio } \\
\text { semanal de } \\
\text { entradas App } \\
3\end{array}$ & 13,5 & 83 & 36,22 & 13,224 & $(19,44-45,89)$ \\
\hline $\begin{array}{c}\text { Promedio } \\
\text { semanal de } \\
\text { entradas a } \\
\text { las } 3 \text { app más } \\
\text { usadas }\end{array}$ & 172,36 & 973,55 & 572,96 & 566,53 & $(6,43-1139,49)$ \\
\hline
\end{tabular}

Como se puede comprobar en la Tabla 5, es innegable que la mayor parte del tiempo empleado en el uso del teléfono móvil se destina a las redes sociales. Cabe destacar, asimismo, la amplia diferencia existente entre los promedios de uso de las 3 aplicaciones más usadas. Por último, es destacable que el alumno que menos tiempo de media está delante de su dispositivo móvil lo usa 873 minutos, más de 14 horas semanales y más de 2 horas diarias.

\subsection{Instrumento 3: Indicadores académicos; calificaciones}

El tercer instrumento empleado fue el registro de las calificaciones académicas del primer trimestre del curso 2019-2020, concretadas en la media aritmética de todas las asignaturas que arroja el programa de gestión académica Plumier XXI de la Consejería de Educación de la Región de Murcia. A partir de estas, se confeccionaron las siguientes figuras (Figura 15 y Figura 16) en las que se muestra la comparación entre tres variables: la nota media del primer trimestre del curso 2019/2020, el IPN y el número de asignaturas suspensas (si las hay). 


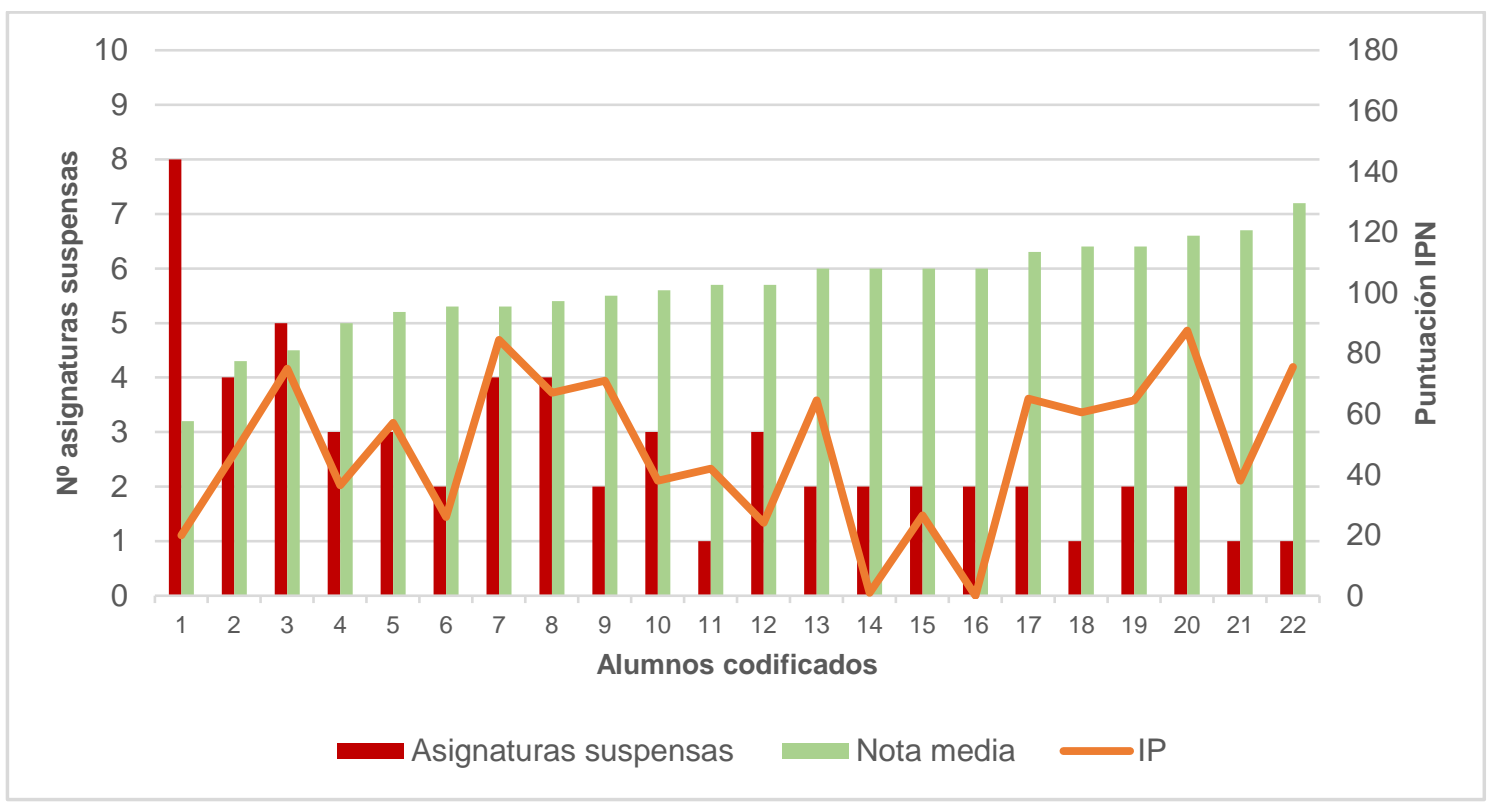

Figura 15. Submuestra alumnos con algún suspenso.

La Figura 15 muestra los datos de los participantes con al menos una asignatura suspensa en el trimestre estudiado $(n=22)$. Cada una de las columnas muestra los datos de un alumno específico codificado, además, ordenados de forma creciente por nota media. En el caso del IPN, la senda es completamente errática, sin ningún patrón; lo que se confirma con los coeficientes de correlación calculados entre el IPN y las otras dos variables, que son prácticamente nulos.

La Figura 16, por su parte, muestra únicamente dos de las tres variables, ya que se incluyen datos de alumnos que carecen de asignaturas con una media por debajo de cinco $(n=30)$. Se puede observar de nuevo con notable nitidez que, al ordenar el alumnado por nota media, dando evidentemente una senda creciente, el IPN vuelve a ser errático, y de nuevo el coeficiente de correlación es prácticamente 0 , hecho que queda visualmente representado en el gráfico.

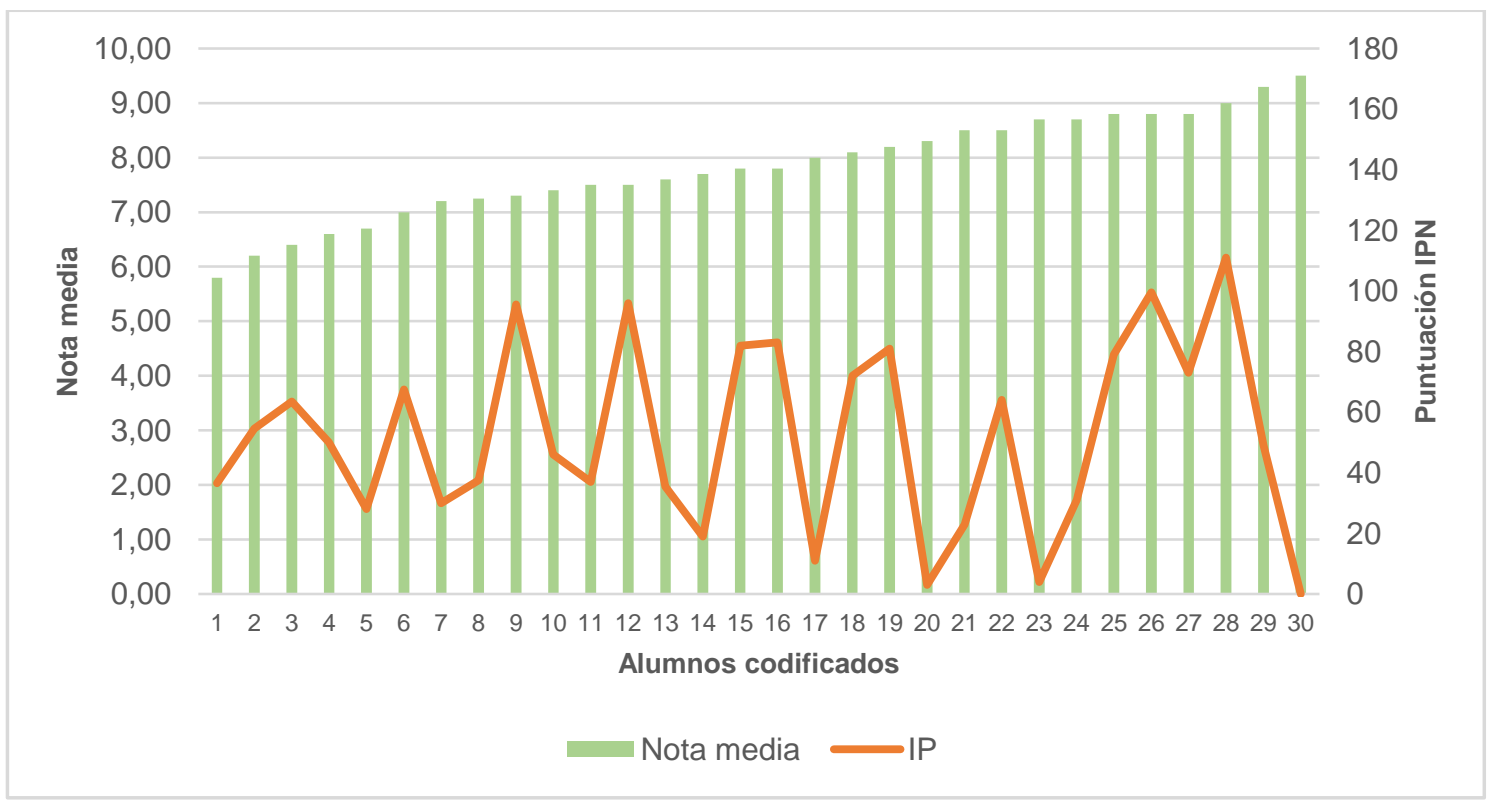

Figura 16. Submuestra aprobados 
Finalmente, es necesario cerrar el análisis estadístico con el estudio de la correlación entre las variables paradigmáticas del mismo. La comparación entre los valores totales de consulta semanal y el IPN devuelven un coeficiente de correlación negativo muy cercano a 0 .

Con el fin de reforzar este dato, se calcularon seis funciones de regresión (Tabla 6):

Tabla 6. Funciones de Regresión

\begin{tabular}{ccc}
\hline Tipo de función & Función & Coeficiente de determinación \\
\hline Exponencial & $y=138,34 \mathrm{e}^{-0,002 x}$ & $\mathrm{R}^{2}=0,0925$ \\
Lineal & $\mathrm{y}=-0,1336 \mathrm{x}+130,57$ & $\mathrm{R}^{2}=0,108$ \\
Logarítmica & $\mathrm{y}=-43,64 \ln (\mathrm{x})+338,44$ & $\mathrm{R}^{2}=0,1195$ \\
Potencial & $\mathrm{y}=1710,2 \mathrm{x}^{-0,528}$ & $\mathrm{R}^{2}=0,1018$ \\
$\begin{array}{c}\text { Polinómica de } \\
\text { grado 2 }\end{array}$ & $\mathrm{y}=0,0007 \mathrm{x}^{2}-0,6216 \mathrm{x}+206,72$ & $\mathrm{R}^{2}=0,1331$ \\
$\begin{array}{c}\text { Polinómica de } \\
\text { grado 3 }\end{array}$ & $\mathrm{y}=2 \mathrm{E}-06 \mathrm{x}^{3}-0,0008 \mathrm{x}^{2}-0,1402 \mathrm{x}+158,65$ & $\mathrm{R}^{2}=0,1341$ \\
\hline
\end{tabular}

Así, se representa gráficamente la función polinómica de grado 3 (Figura 17), que, con un valor de 0,13 , es la que cuenta con mayor coeficiente de determinación y aun así no resulta en absoluto representativa de la distribución de la nube de puntos.

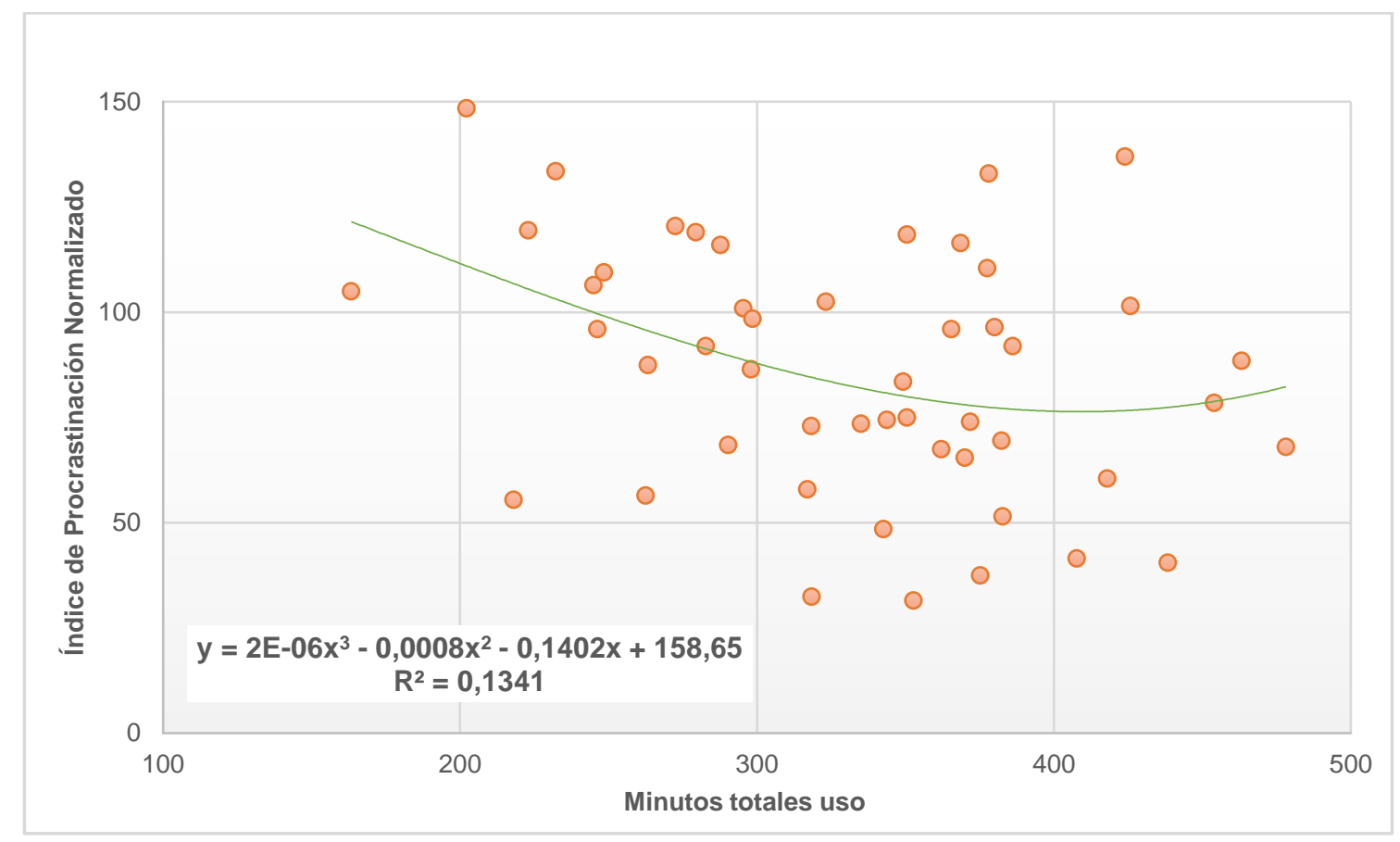

Figura 17. Función de regresión

Aludiendo, por un lado, al coeficiente de correlación muy próximo a 0 y, por otro, a los coeficientes de determinación prácticamente nulos, y por ello, con una pésima bondad de ajuste en todas las funciones estudiadas, resulta evidente la inexistencia de relación estadística y funcional de ambos parámetros.

Además, aunque el signo en estos valores de correlación carece de significación, ni siquiera en estos niveles se atisban movimientos variables positivos; al contrario, si los hubiera, dichos movimientos serían opuestos, es decir, a más tiempo de uso, menos procrastinación. 


\section{CONCLUSIONES}

El objetivo general de esta investigación consiste en comprobar el nivel de tecnoprocrastinación en estudiantes de enseñanza secundaria y universitaria a partir de una hipótesis de partida, que consistía en saber si realmente el uso excesivo de los teléfonos móviles afectaba al rendimiento y las calificaciones académicas. Para ello, seleccionamos dos objetivos específicos con los que responder a ambas cuestiones.

\subsection{Objetivo 1: Analizar el grado de procrastinación existente en el alumnado de enseñanzas medias y superiores}

Para alcanzar este objetivo, utilizamos el cálculo del IPN sobre ambas muestras, cuya fórmula ya ha sido explicada en apartados anteriores.

Tras analizarla, aplicar el test de normalidad, representarla gráficamente y obtener las medidas de posición central y dispersión, se observa que el IPN sigue una Distribución Normal con media 88 y desviación típica 32, quedando así la muestra dividida en dos mitades prácticamente iguales; con lo que se concluye, por tanto, que los alumnos que procrastinan más son prácticamente los mismos que los que no, considerando la media del IPN (88), el valor límite para definir procrastinador, o no, el comportamiento del estudiante. Dicha estructura refuerza la validez del IPN al replicar fielmente la distribución normal descrita.

\subsection{Objetivo 2: Establecer una correlación entre el grado de procrastinación del alumnado provocado por el uso de dispositivos móviles, y sus calificaciones}

Para este objetivo, se presenta una tabla (Tabla 7) y una figura como resumen de las correlaciones obtenidas:

Tabla 7. Correlaciones

\begin{tabular}{cc}
\hline Variables & Coeficiente de correlación \\
\hline Minutos totales semanales en RRSS - IPN & $-0,165$ \\
Minutos totales semanales en RRSS - & 0,076 \\
Calificaciones & \\
Minutos totales semanales de uso - IPN & $-0,279$ \\
Minutos totales semanales de uso - \\
$\quad$ Calificaciones \\
Calificaciones - IPN
\end{tabular}

Todos los valores del coeficiente de correlación representados en la Tabla 7 refleja que no existe una correlación significativa entre ninguno de los pares de variables, ya que para que se considere esa existencia de correlación significativa es preciso superar en valor absoluto 0,7 (positivo o negativa). Los resultados muestran en todos los casos valores absolutos inferiores a 0,3, por tanto, no existe ningún tipo de correlación entre los pares de variables estudiados.

La hipótesis de partida suponía que el uso excesivo del teléfono móvil (tecnoprocrastinación) afectaría de manera significativa a las calificaciones académicas. Sin embargo, el estudio no apoya la misma, y, por tanto, la procrastinación y el rendimiento académico, en el contexto de este trabajo, no dependen de ninguna manera del tiempo de uso que se dé al teléfono móvil, por lo que habría que buscar su origen en otro tipo de factores, ya sea la gestión del tiempo, la organización del trabajo individual u otros.

\section{DISCUSIÓN}

Son abundantes los estudios que relacionan la procrastinación académica con factores que pueden ser relevantes en su desarrollo. Entre ellos se han destacado características de la personalidad (Ackerman \& Gross, 2005) o la autorregulación, la autoeficacia académica y la 
autoestima (Klassen et al. 2008). En los últimos años, se ha producido un aumento de la presencia de las TIC en el aula a consecuencia de la era de la hiperconectividad en la que vivimos (Pinto \& Díaz, 2015), subrayando Castro \& Mahamud (2017) que esta sobreexplotación de las TIC puede ser perjudicial para el progreso de los adolescentes en el entorno académico si no se utilizan de manera adecuada.

A pesar de no ser una línea tratada ampliamente en la literatura científica, como ya se ha puntualizado anteriormente, existen estudios que afirman la existencia de una correlación positiva y significativa entre la procrastinación académica y la adicción a internet (Castro \& Mahamud, 2017) o las redes sociales (Matalinares et al., 2017; Núñez-Guzmán \& CisnerosChávez, 2019; Mamani \& Gonzales, 2019). Guevara \& Contreras (2019) defienden que, en el ámbito universitario, el mayor uso del móvil provoca un incremento que posterguen con mayor frecuencia sus asuntos académicos.

Por su parte, el estudio de García \& Zecenarro (2019) arroja datos en los que el alumnado no presenta niveles significativos de correlación entre la adicción a internet, a las redes sociales y la dependencia al móvil con respecto a la procrastinación académica, coincidiendo con los resultados del presente trabajo.

Es significativo, por tanto, que los resultados presentados en este documento cuestionan y ponen en duda la relación directa entre el uso de dispositivos móviles y la mala gestión del tiempo, quedando así abierta una posible vía de comprobación de las condiciones, edades o niveles en los que existe un uso de estos dispositivos que perjudica el desarrollo académico.

En cuanto a limitaciones y posibles líneas futuras de investigación, se podría partir de una muestra más representativa, centrada en el alumnado de España, incluso separado por niveles educativos, y no solo para identificar las causas de esta ausencia de correlación, sino también para profundizar sobre el origen de la procrastinación en el ámbito académico.

\section{ENLACES}

Instrumentos y datos de investigación: https://is.gd/ynsdvN

\section{REFERENCIAS BIBLIOGRÁFICAS}

Ackerman, D.S. \& Gross, B.L. (2005). My instructor made me do it: Task characteristics of procrastination. Journal of Marketing education, 27(1), 5-13. https://bit.ly/3bDI9dL

Álvarez, B.O. (2010). Procrastinación general y académica en una muestra de estudiantes de secundaria de Lima metropolitana. Persona, 13, 159-177. http://dx.doi.org/10.26439/persona2010.n013.270

Angarita, L.D. (2012). Aproximación a un concepto actualizado de la procrastinación. Revista Iberoamericana de Psicología: ciencia y tecnología, 5(2), 85-94. https://doi.org/10.33881/2027-1786.rip.5209

Castro, B. \& Mahamud, R. (2017). Procrastinación académica y adicción a internet en estudiantes universitarios de Lima Metropolitana. https://bit.ly/3fOLidJ

Cano, C. A. G., Palomá, N. F. O. \& Rojas, L. M. P. (2016). Procrastinación y factores relacionados para su análisis en la educación superior. I+ $D$ Revista de Investigaciones, 7(1), 32-39. https://doi.org/10.33304/revinv.v07n1-2016004

Chu, A. H. C. \& Choi, J. N. (2005). Rethinking procrastination: Positive effects of" active" procrastination behavior on attitudes and performance. The Journal of social psychology, 145(3), 245-264. https://doi.org/10.3200/SOCP.145.3.245-264

Dewitte, S. \& Schouwenburg, H. C. (2002). Procrastination, temptations, and incentives: The struggle between the present and the future in procrastinators and the punctual. European Journal of personality, 16(6), 469-489. https://doi.org/10.1002/per.461 
Ferrari, J. R. (2000). Procrastination and attention: Factor analysis of attention deficit, boredomness, intelligence, self-esteem, and task delay frequencies. Journal of Social Behavior and Personality, 15(5), 185-196. https://bit.ly/3u2TIII

García, D. \& Zecenarro, L. A. (2019). Adicción a internet, Adicción a las Redes Sociales, Dependencia al Móvil y su influencia sobre la Procrastinación Académica en una muestra de Adolescentes de la ciudad de Juliaca [Trabajo Fin de Grado, Universidad Peruana Unión]. Repositorio UPEU. https://bit.ly/2RwGH67

Guevara, G. C. \& Contreras, A. T. (2019). Relación entre dependencia al teléfono móvil y procrastinación en estudiantes de una universidad privada de Lima Este [Trabajo Fin de Grado, Universidad Peruana Unión]. Repositorio UPEU. https://bit.ly/3hDIzor

Klassen, R. M., Krawchuk, L. L. \& Rajani, S. (2008). Academic procrastination of undergraduates: Low self-efficacy to self-regulate predicts higher levels of procrastination. Contemporary Educational Psychology, 33(4), 915-931. https://doi.org/10.1016/i.cedpsych.2007.07.001

Mamani, A. L. \& Gonzales, D. (2019) Adicción a redes sociales y su relación con la procrastinación académica en estudiantes de nivel secundario de una Institución Educativa Privada en Lima-Este [Trabajo Fin de Grado, Universidad Peruana Unión]. Repositorio UPEU. https://bit.ly/3f1n1QV

Matalinares, M. L., Diaz, A. G., Rivas, L. H., Dioses, A. S., Arenas, C. A., Raymundo, O., Baca, D., Uceda, J., Yaringaño, J., \& Fernandez, E. (2017). Procrastinación y adicción a redes sociales en estudiantes universitarios de pre y post grado de Lima. Horizonte De La Ciencia, 7(13), 63-81. https://bit.ly/3bFkcTr

Núñez-Guzmán, R., \& Cisneros-Chávez, B. C. (2019). Adicción a Redes Sociales y Procrastinación Académica en Estudiantes Universitarios. Editor. Nuevas ldeas en Informática Educativa, 15, 114-120. https://bit.ly/3bDWK90

Pinto, A. R., \& Díaz, J. D. (2015). Convivencia Escolar en la era de la hiperconectividad. Cultura Educación y Sociedad, 6(1), 149-164. https://bit.ly/3v1a8xR

Quan-Haase, A. \& Wellman, B. (2005). Local virtuality in an organization: Implications for community of practice. In P. Van Den Besselaar, G. De Michelis, J. Preece \& C. Simone (Eds.), Communities and technologies 2005 (pp. 215-238). Springer. https://doi.org/10.1007/1-4020$\underline{3591-8 \quad 12}$

Reig, D. \& Vílchez L. (2013). Los jóvenes en la era de la hiperconectividad: tendencias, claves y miradas. Madrid: Fundación Telefónica y Fundación Encuentro. REDEX: Revista Educativa de Extremadura, 3(5), 212. http://dx.doi.org/10.12795/revistafuentes

Robert, P. (1972). S.N.L. Société du Nouveau Littré, Dictionnaire alphabétique et analogique de la langue française, Le Petit Robert, Dictionnaire Le Robert, Paris. Société du Nouveau.

Rodríguez, A., \& Clariana, M. (2017). Procrastinación en estudiantes universitarios: su relación con la edad y el curso académico. Revista Colombiana de Psicología, 26(1), 45-60. http://dx.doi.org/10.15446/rcp.v26n1.53572

Rosario, P., Costa, M., Nuñez, J. C., González-Pienda, J., Solano, P. \& Valle, A. (2009). Academic procrastination: Associations with personal, school, and family variables. The Spanish Journal of Psychology, 12(1), 118-127. https://bit.ly/3bWh3iv

Senecal, C., Koestner, R. \& Vallerand, R. J. (1995). Self-regulation and academic procrastination. The journal of social psychology, 135(5), 607-619. https://doi.org/10.1080/00224545.1995.9712234

Solomon, L.J. \& Rothblum, E.D. (1994). Procrastination assessment scale-students (pass). In J. Fischer and K. Corcoran (Eds.), Measures for clinical practice (pp. 446-452). The Free Press.

Steel, P. (2007). The nature of procrastination: A meta-analytic and theoretical review of quintessential self-regulatory failure. Psychological bulletin, 133(1), 65. https://doi.org/10.1037/0033-2909.133.1.65 
Ugaz, Y. \& Lizana, D.P. (2019) Procrastinación académica y dependencia al móvil en los estudiantes del 4to y 5to grado del nivel secundario de la Institución Educativa Emblemático Serafín Filomeno, Moyobamba [Trabajo Fin de Grado, Universidad Peruana Unión]. Repositorio UPEU. https://bit.ly/33Xic4J

Uzun, B., O'Callaghan, J., Bokszczanin, A., Ederer, E. \& Essau, C. (2014). Dynamic interplay of depression, perfectionism and self-regulation on procrastination. British Journal of Guidance \& Counselling, 42(3), 309-319. https://doi.org/10.1080/03069885.2014.896454

Toro, J. (2010). El adolescente en su mundo. Riesgos, problemas y Trastornos. Pirámide.

\section{INFORMACIÓN SOBRE LOS AUTORES}

\section{Cristina Ramón García \\ Universidad Miguel Hernández}

Estudiante de Medicina en la Universidad Miguel Hernández y Matrícula de Honor en Bachillerato de Ciencias, Investigación Plurilingüe, LOMCE, IES Los Albares, 2020. Nivel B2 de inglés (Cambridge) y B1 de francés a través de la Escuela Oficial de Idiomas. Participación en el Programa Campus Científicos de Verano del Ministerio de Educación, Cultura y deporte, y la FECYT, 2018; tercer Premio Nacional "El País de los Estudiantes" del Periódico "El País", 2019 y participante en Estancia formativa en el extranjero (Irlanda), Beca de la Consejería de Educación, Juventud y Deportes Región de Murcia, 2019.

\section{Pedro Peinado Rocamora \\ Universidad Internacional de La Rioja}

Licenciado en Administración y Dirección de Empresas UMU (2003); doctor en Tecnología Educativa UMU (2018) "La Clase Invertida: Una experiencia con alumnos con dificultades de aprendizaje"; Master MBA. ENAE (2003); Máster en e-learning y redes sociales por la UNIR (2014). Actualmente profesor de matemáticas (CARM) y profesor contratado en la UNIR (Máster de educación).

Premio Nacional a la Calidad Educativa Giner de los Ríos (MECD Y FUNDACIÓN BBVA, 2017), Premio Regional Innovación Educativa Docente (CONSEJERÍA DE EDUCACIÓN CARM, 2017), Premio Nacional de Elaboración de Recursos Digitales (MECD, 2015). Ponente en las Jornadas Nacionales del Aprendizaje de las Matemáticas (Cartagena, 2015) y participante en mesa redonda del VII Seminario Interuniversitario de Investigación en Tecnología Educativa (Lérida, 2020). Publicaciones en diversas revistas: Las Pizarras Digitales Interactivas desde el punto de vista del alumno (Revista TIC en las aulas, 2013); aumento de la motivación mediante el uso de redes sociales (En DIM: Revista Didáctica, Innovación y Multimedia, 2014); La Clase Invertida: revisión sistemática en el periodo 2010-2017 (Revista Docencia e Investigación, 2018) y La Clase Invertida: Una experiencia con alumnos con dificultades de aprendizaje (Revista Edutec, 2019).

\section{María Elena Rodríguez Nieto}

Consejería de Educación de la Región de Murcia

Licenciada Filología Románica (francés) por la Universidad de Extremadura (1986) y D.E.A. en Estudios Románicos por la Universidad de París-Sorbona (1992). Actualmente profesora de francés (CARM), correctora examinadora oficial de la Alianza Francesa por el Ministerio de Educación francés y Tutora de prácticas curriculares Máster Formación Profesorado ESO, Bachillerato, FP y Enseñanza de Idiomas (UMU).

Publicaciones en el Diccionario Larousse en 2004, 2016 y 2018. Participante en diversos talleres de difusión científica como en las Jornadas de la enseñanza bilingüe en Murcia (2016), en las Jornadas Uso responsable y seguro de Internet y redes sociales en el aula en Murcia (2017) o el Proyecto de innovación PROMECE: mejora del aprendizaje del alumnado, colaboración centros públicos ámbito nacional, Instituto Nacional de Tecnologías Educativas y de Formación del Profesorado, Ministerio de Educación, Cultura y Deporte (2017).

Los textos publicados en esta revista están sujetos a una licencia de Reconocimiento 4.0 España de Creative Commons. Puede copiarlos, distribuirlos, comunicarlos públicamente y hacer obras derivadas siempre que reconozca los créditos de las obras (autoría, nombre de la revista, institución editora) de la manera especificada por los autores o por la revista. La licencia completa se puede consultar en:Licencia Creative Commons Atribución-NoComercial-Compartir por igual 4.0 Internacional. 